\title{
A Giant Abdominal Aortic Aneurysm and a Massive Eye Hemorrhage Due to Advanced Atherosclerosis in an Eighty-Four Year Old Man
}

\author{
Mohammad Otahbachi ${ }^{1}$, Cihan Cevik*,2 ${ }^{*}$ David Hodges ${ }^{2}$, Ashwani Kumar ${ }^{1}$ and Leigh Ann Jenkins ${ }^{1}$ \\ ${ }^{I}$ Texas Tech University Health Sciences Center, Cardiology Division, Lubbock, TX, USA, ${ }^{2}$ Texas Tech University Health \\ Sciences Center, Department of Internal Medicine, Lubbock, TX, USA
}

Texas Tech University Health Sciences Center, School of Medicine, Department of Internal Medicine, Lubbock, TX, USA 79430

Keywords: Aneurysm, abdominal aorta, atherosclerosis.

\section{INTRODUCTION}

An 84-year-old man presented to the ophthalmology outpatient clinic complaining of decreased vision in his left eye. His past medical history included hypertension and dyslipidemia. He did not have diabetes and he was not a smoker. Eye examination revealed hemorrhagic retinal detachment (Fig. 1). This abnormality can be secondary to metastatic tumors, therefore abdominal and chest CT scans were ordered. $\mathrm{CT}$ of the abdomen demonstrated a huge infrarenal abdominal aortic aneurysm (AAA), extending from the level of the first lumbar vertebrae to the second sacral vertebrae. The aneurysm was measured $9.4 \mathrm{~cm}$. in length and $15.1 \mathrm{~cm}$. in maximal transverse diameter (Fig. 2). The aneurysm was initially diagnosed six years ago, but the patient had refused surgery or endovascular repair at that time. The maximal transverse diameter was measured as 6.5 $\mathrm{cm}$ at his first diagnosis in 2003. After declining further work-up, the patient underwent uneventful eye surgery under local anesthesia. Unfortunately, he expired soon after discharge from the hospital secondary to rupture of the aneurysm.

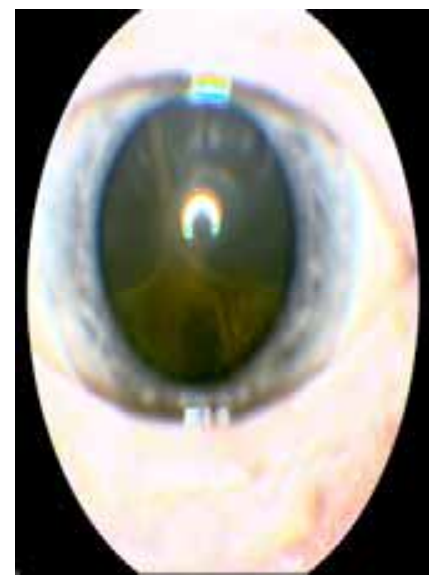

Fig. (1). Large hemorrhagic-exudative choroidal and retinal detachment.

Address correspondence to this author at the Texas Tech University Health Sciences Center, Cardiology Department, $36014^{\text {th }}$ Street, Lubbock, TX, 79430, USA; Tel: 1-806-7433155; Fax: 1-806-7433148;

E-mail: cihan.cevik@ttuhsc.edu

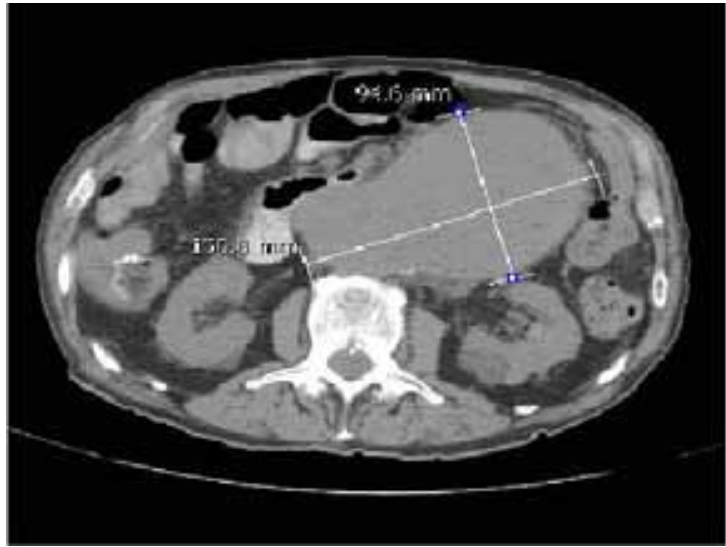

Fig. (2). Abdominal computed tomography: A giant abdominal aortic aneurysm, maximal diameter $15.1 \mathrm{~cm}$.

Abdominal aortic aneurysm (AAA) is defined as the dilatation to more than one and a half times the normal diameter of the aorta. The incidence is approximately nine times higher in males than females. AAA is more common than thoracic aneurysms and the majority of cases involve the infra-renal aorta. AAA generally enlarges at a rate of 0.4 $\mathrm{cm} /$ year; however, the rate of expansion is variable among populations and sometimes within the same patient. Larger aneurysms tend to enlarge faster following Laplace's law, in which wall tension is a product of internal pressure and vessel radius. Thus, as aneurysm diameter increases, wall tension increases as well, leading to further increase in the diameter, the so-called vicious cycle of aneurysm growth.

Massive intraocular hemorrhage occurs in patients with macular degeneration, high blood pressure, diabetes, advanced age, anticoagulant medications, and idiopathic thrombocytopenic purpura [1, 2]. Arteriosclerotic vasculature causes fluctuations in the introcular pressure as well as elevated systemic blood pressure which, in turn, facilitates intraocular bleeding. We report a patient with a giant infrarenal abdominal aortic aneurysm and large hemorrhagicexudative choroidal and retinal detachment. Both conditions occur in individuals with history of advanced atherosclerosis and cardiovascular diseases. Our case represents one of the largest abdominal aortic aneurysm ever reported. 


\section{REFERENCES}

[1] Tilanus MA, Vaandrager W, Cuypers MH, Verbeek AM, Hoyng CB. Relationship between anticoagulant medication and massive intraocular hemorrhage in age-related macular degeneration. Graefes Arch Clin Exp Ophthalmol 2000; 238: 482-5.
[2] Okuda A, Inoue $M$, Shinoda K, Tsubota K. Massive bilateral vitreoretinal hemorrhage in patient with chronic refractory idiopathic thrombocytopenic purpura. Graefes Arch Clin Exp Ophthalmol 2005; 243: 1190-3.

(C) Otahbachi et al.; Licensee Bentham Open.

This is an open access article licensed under the terms of the Creative Commons Attribution Non-Commercial License (http: //creativecommons.org/licenses/by$\mathrm{nc} / 3.0 /$ ) which permits unrestricted, non-commercial use, distribution and reproduction in any medium, provided the work is properly cited. 